\title{
Inherent Structure Entropy of Supercooled Liquids
}

\author{
F. Sciortino ${ }^{(1)}$, W. Kob ${ }^{(2)}$ and P. Tartaglia(1) \\ (1) Dipartimento di Fisica and Istituto Nazionale per la Fisica della Materia, Universitá di Roma \\ La Sapienza, \\ P.le Aldo Moro 2, I-00185, Roma, Italy \\ (2) Institut für Physik, Johannes Gutenberg-Universität, Staudinger Weg 7, D-55099 Mainz, \\ Germany
}

(April 10, 2018)

\begin{abstract}
We present a quantitative description of the thermodynamics in a supercooled binary Lennard Jones liquid via the evaluation of the degeneracy of the inherent structures, i.e. of the number of potential energy basins in configuration space. We find that for supercooled states, the contribution of the inherent structures to the free energy of the liquid almost completely decouples from the vibrational contribution. An important byproduct of the presented analysis is the determination of the Kauzmann temperature for the studied system. The resulting quantitative picture of the thermodynamics of the inherent structures offers new suggestions for the description of equilibrium and out-of-equilibrium slow-dynamics in liquids below the Mode-Coupling temperature.
\end{abstract}

In recent years, a significant effort has been devoted to understanding the fundamental nature of glass-forming materials, a long-standing open problem in condensed matter physics [1]. Theoretical [2, 4], experimental [5, 6] and numerical efforts [7] have broadened our knowledge of the physical mechanisms responsible for the dramatic slowing down of the dynamics in supercooled liquids (more than 15 order of magnitudes) as the temperature $T$ changes over a small range, as the glass transition temperature is approached.

Some recent theoretical approaches build upon ideas which were presented several decades ago [8 12]. In these works, the slowing down of the dynamics was connected to the presence of basins in configuration space. The short time dynamics (on a ps time scale) was related to the process of exploring a finite region of phase space around a local potential energy minimum, while the long time dynamics was connected to the transition among different local potential energy minima [10,12]. In this picture, upon cooling the intra-basin motion becomes more and more separated in time from the slow (and strongly $T$-dependent) inter-basins motion. The decrease of the entropy of supercooled liquids on cooling 8$]$ was associated with the progressive ordering of the system in configuration space, i.e. in the progressive population of basins with deeper energy but of lower degeneracy [9].

Following these ideas, Stillinger and Weber [12] introduced the concept of inherent structure (IS), defined as local minimum configuration of the $3 N$-dimensional potential energy surface. A basin in configuration space was defined as the set of points that — via a steepest descent path along the potential energy hypersurface - map to the same IS. This precise 
operational definition of a basin allows the configuration space to be partitioned into an ensemble of distinct basins. Thus, the canonical partition function $Z_{N}$ for a system of $N$ atoms at inverse temperature $\beta=1 / k_{B} T$ can be written as

$$
Z_{N}=\lambda^{-3 N} \sum_{\alpha} \exp \left(-\beta \Phi_{\alpha}\right) \int_{R_{\alpha}} \exp \left[-\beta \Delta_{\alpha}\left(\mathbf{r}^{N}\right)\right] d \mathbf{r}^{N}
$$

where $R_{\alpha}$ is the set of points composing the basin $\alpha, \Phi_{\alpha}$ is the potential energy of minimum $\alpha$ and the non-negative quantity $\Delta_{\alpha}\left(\mathbf{r}^{N}\right)$ measures the potential energy at a point $\mathbf{r}^{N}$ belonging to the basin $\alpha$ relative to the minimum. The integration over the momenta introduces the thermal wavelength $\lambda=\sqrt{\beta h^{2} / 2 \pi m}$, where $m$ is the mass. Eq. (1) shows that both the IS energy and the thermal excitation within the basin region $R_{\alpha}$ contribute to $Z_{N}$. Stillinger and Weber also noted that, if the value of the potential energy minimum uniquely characterizes the properties of the basin, then a very powerful simplification of Eq. (1) can be performed. By introducing a density of states $\Omega\left(e_{I S}\right)$ with IS-energy $e_{I S}, Z_{N}$ can be written as

$$
Z_{N} \approx \int d e_{I S} \Omega\left(e_{I S}\right) \exp \left[-\beta e_{I S}-\beta f\left(\beta, e_{I S}\right)\right]
$$

where

$$
-\beta f\left(\beta, e_{I S}\right)=\ln \left(\int_{R\left(e_{I S}\right)} \exp \left[-\beta \Delta_{e_{I S}} \Phi\left(\mathbf{r}^{N}\right)\right] \frac{d \mathbf{r}^{N}}{\lambda^{3 N}}\right)
$$

can be interpreted as free energy of the system when confined in one of the characteristic basins with IS energy $e_{I S}$. Then, the probability that a configuration of the liquid extracted from an equilibrium ensemble of configurations at temperature $T$ is associated to an IS with energy between $e_{I S}$ and $e_{I S}+\delta e_{I S}$ is

$$
P\left(e_{I S}, T\right)=\frac{\Omega\left(e_{I S}\right) \delta e_{I S} \exp \left[-\beta e_{I S}-\beta f\left(\beta, e_{I S}\right)\right]}{Z_{N}(\beta)}=\frac{\exp \left[-\beta\left(e_{I S}-T S_{\text {conf }}\left(e_{I S}\right)+f\left(\beta, e_{I S}\right)\right)\right]}{Z_{N}(\beta)}
$$

where we have defined $S_{\text {conf }}\left(e_{I S}\right) \equiv k_{B} \ln \left[\Omega\left(e_{I S}\right) \delta e_{I S}\right]$, since $\Omega\left(e_{I S}\right) \delta e_{I S}$ is the number of states between $e_{I S}$ and $e_{I S}+\delta e_{I S}$.

The formalism proposed by Stillinger and Weber, although often used in the past to clarify structural issues in liquids [13, 14], for long time was not quantitatively applied to computer studies of the glass-transition problem, due to the significant computational effort required in equilibrating atomic configurations at low $T$. Only recently, Sastry et al 15 addressed the problem of evaluating the $T$ dependence of the average IS energy $\left(\bar{e}_{I S}\right)$ in supercooled states in a binary mixture of LJ particles, observing a significant decrease of $\bar{e}_{I S}$ on supercooling. This result, also observed for other models for liquids, e.g. in models for water [14] and orthoterphenyl [16], furnishes strong evidence of the relevant role played by the low-energy basins on cooling. In a recent work [17], we proposed to invert the relation between the $\bar{e}_{I S}$ energy and $T$ to define an effective temperature at which the configurational part of the system is in equilibrium. This hypothesis, which has been proven useful in interpreting the aging process in a model liquid in terms of progressive thermalization of the IS [17], support the validity of Eq. (2) and together with the work of Sastry et al., calls 
for an effort in the direction of checking the formal expression for the supercooled liquid free energy, i.e. the $T$-range of validity, and an effort in the direction of evaluating the $e_{I S}$ dependence of the configurational entropy. This article is a first effort in this direction.

The model system we study is the well-known 80-20 Lennard Jones $A-B$ binary mixture [18], composed by a 1000 atoms in a volume $V_{o}=(9.4)^{3}$, corresponding to a reduced density of 1.2039. Units of length and energy are defined by the $\sigma$ and $\epsilon$ parameters of the $A-A$ Lennard Jones interaction potential. The mass of atom $A$ is chosen to be 1 . In these units, $k_{B}=1$. Simulations, covering the range $0.446<T<5$, have been performed in the canonical ensemble by coupling the system to a Nose'-Hoover thermostat [19]. This system is well characterized and its slow dynamics has been studied extensively [18]. The critical temperature of Mode Coupling Theory $T_{M C T}$ for this system is 0.435 [18].

Between 500 and 1000 equilibrium configurations for each $T$ (covering more than 80 millions integration time steps for each $T$ ) have been quenched to their local minima by using a standard conjugate-gradient minimization algorithm. By performing this large number of quenches we are able to determine not only $\bar{e}_{I S}$ and its $T$ dependence but also the probability distribution $P\left(e_{I S}, T\right)$, shown in Fig.가 $(\mathrm{A})$.

In the $T$-region where Eq. (田) is supposed to hold, curves of $\ln \left[P\left(e_{I S}, T\right)\right]+\beta e_{I S}$ are equal to $S_{\text {conf }}\left(e_{I S}\right) / k_{B}-\beta f\left(\beta, e_{I S}\right)$, except for the $T$-dependent constant $\ln [Z(\beta)]$. If $f\left(\beta, e_{I S}\right)$ has only a weak dependence on $e_{I S}$, then it is possible to superimpose $P\left(e_{I S}, T\right)$ curves at different temperatures which overlap in $e_{I S}$. The resulting $e_{I S}$-dependent curve is, except for an unknown constant, $S_{\text {conf }}\left(e_{I S}\right) / k_{B}$ in the $e_{I S}$ range sampled within the studied $T$ interval. This procedure is displayed in Fig.1-(B). We note that while below $T=0.8$ curves for different $T$ lie on the same master curve, above $T=0.8$, curves for different $T$ have different $e_{I S}$ dependence, thus showing the progressive $e_{I S}$-dependence of $f\left(\beta, e_{I S}\right)$. The overlap between different $P\left(e_{I S}, T\right)$ curves below $T=0.8$ indicates that the obtained master curve is indeed, except for an unknown constant, the $e_{I S}$ configurational entropy, i.e. the logarithm of the number of basins with the same $e_{I S}$ value.

It is particularly relevant that, for $T<0.8, f\left(\beta, e_{I S}\right) \approx f(\beta)$ and thus $Z_{N}$ (Eq. 2) is well approximated by the product of a vibrational contribution $\left[e^{-\beta f}\right]$ and of a configurational contribution depending only on the IS-energies and their degeneracy $\left[\int d e_{I S} \Omega\left(e_{I S}\right) e^{-\beta e_{I S}}\right]$. Thus the liquid can be considered as composed by two independent subsystems, respectively described by the IS and by the vibrational part. The IS subsystem can be considered as a continuum of levels characterized by an energy value $e_{I S}$ and an associated degeneracy $\Omega\left(e_{I S}\right)$. When the IS-subsystem is in thermal equilibrium (with the vibrational subsystem), then the $T$ dependence of the average configurational entropy $\bar{S}_{\text {conf }}$ can be evaluated using the standard thermodynamic relation

$$
\frac{d \bar{S}_{\text {conf }}(T)}{d \bar{e}_{I S}}=\frac{1}{T}
$$

i.e. by integrating the $T$ dependence of $d \bar{e}_{I S} / T$.

While the evaluation of the $T$-dependence of $S_{\text {conf }}$ already furnishes relevant information, the evaluation of the unknown integration constant would allow for a determination of the number of IS with the same $e_{I S}$ and, via a suitable low $T$ extrapolation, to the determination of the so-called Kauzmann temperature $T_{K}$, i.e. the $T$ at which the configurational entropy appears to approach zero. To do so we exploit the fact that Eq. 2 predicts that the liquid free energy $F_{\text {liquid }}(T)$ can be written as 20] 


$$
F_{\text {liquid }}(T)=-k_{B} T \ln \left[Z_{N}(T)\right]=\bar{e}_{I S}(T)-T S_{\text {conf }}\left(\bar{e}_{I S}(T)\right)+f\left(\beta, \bar{e}_{I S}(T)\right)
$$

where $\bar{e}_{I S}(T)$ is the $e_{I S}$ value which maximises the integrand in Eq. (2). We assume that at the lowest studied $T$, the unknown $f\left(\beta, \bar{e}_{I S}\right)$ can be approximated by the harmonic free energy of a disordered system characterized by the eigenfrequencies spectrum calculated from the distribution of IS at the corresponding $T$ [21]. In this approximation, the entropy of the liquid, which can be calculate via thermodynamic integration, once the entropy of the corresponding harmonic disordered solid is subtracted, provides an estimate of the configurational entropy in absolute units.

To calculate the liquid entropy we perform a thermodynamic integration first along the $T=5.0$ isotherm, from infinite volume down to $V_{o}$, followed by a $T$ integration of the specific heat at fixed volume, down to the lowest studied temperature. Since the $T$ dependence of the potential energy $E$ along the studied isochore is extremely well described by the law $E(T) \sim T^{3 / 5}$, in agreement with recent theoretical predictions for dense fluids [22], the potential energy contribution to the liquid entropy follows the law $T^{-2 / 5}$. By summing the kinetic energy contribution we obtain an analytic expression which can reliably be extrapolated to temperatures lower than the studied ones.

The evaluated $T$ dependence of liquid and disordered-solid entropies is reported in Fig.2. We also show in Fig. 2 the contribution to the disordered-solid entropy arising from the $T$-dependence of the eigenfrequencies spectrum, to confirm that the $T$-dependence of the harmonic solid frequencies contribute only weakly to the entropy. The $T$ dependence of $\Delta S \equiv S_{\text {liquid }} S_{\text {disordered-solid }}$ is shown in Fig. 3-(A). We note that this difference vanishes at $T=0.297 \pm 0.02$, which defines $T_{K}$ for the studied binary mixture. An independent recent estimate of $T_{K}$ for this system, based on an integral equation approach and on a similar analysis of simulation data, is $T_{K}=0.29$ [22]. The resulting ratio between $T_{K}$ and $T_{M C T}$ support the view that the studied system has intermediate fragility character, as recently predicted by Angell and coworkers on the basis of a comparison between experimental results and numerical data for the same system [24].

At the lowest studied temperatures, where the harmonic approximation is valid [21], $\Delta S$ coincides with $S_{\text {conf }}(T)$. We use this identity to calculate the unknown integration constant for the inherent structure entropy. Moreover, by integrating $\operatorname{Td}(\Delta S(T))$, both the configurational energy dependence of the configurational entropy (Fig. 且-(c)) and the $T$ dependence of the configurational energy (3t(b)) can be evaluated, allowing to bridge the gap between $T_{K}$ and the lowest $T$ at which we were able to equilibrate the system. The present analysis predicts $e_{I S}\left(T_{K}\right)=-7.82 \pm 0.01$. Thus, both the configuration entropy and energy around $T_{M C T}$ is halfway between $T_{K}$ and the high $T$ value, suggesting that the ordering process in configuration space at the lowest temperature which we have been able to equilibrate is far from being complete.

The data reported in this article offer a quantitative thermodynamic analysis of the supercooling state. This picture confirms the fruitful ideas put forward long time ago [8 [12] and shows that a thermodynamic approach for the inherent structures subsystem becomes possible in supercooled states, since the thermodynamics of the inherent structures almost completely decouples from the "vibrational" thermodynamics. In particular, the quantitative evaluation of the degeneracy of the inherent structures for a well characterized system constitutes a basis for a comprehensive description of the slow dynamics below $T_{M C T}$, the $T$-range in which an accurate theoretical prediction is still missing. Previous estimates of 
the degeneracy of $e_{I S}$, the key ingredient for a descriptions of the dynamics in terms of IS, were available only for systems composed by less than 50 atoms [25,26]. The proposed view of a supercooled liquids as composed by two weakly coupled subsystems - the IS subsystem and the "vibrational" subsystem — will also offer stimulating ideas for a microscopic understanding both of the out-of-equilibrium thermodynamics theories recently proposed [27] and of the aging process [17,28].

Acknowledgment: We thank B. Coluzzi, G. Parisi and P. Verrocchio for sharing with us their recent independent results of the BM LJ system ( [23]). We thank G. Parisi for bringing to our attention Ref. [22]. We thank S. Ciuchi, A. Crisanti, K. Kawasaki, E. La Nave, P. Poole, A. Scala for comments. F.S. and P.T. acknowledge support from MURST PRIN 98 and W.K. from the DFG through SFB 262. 


\section{REFERENCES}

[1] For recent review see articles in Science 267, (1995) and M.D. Ediger, C.A. Angell, and S.R. Nagel, J. Phys. Chem 100, 13200 (1996).

[2] W. Götze, J. Phys.: Condens. Matter 11, A1 (1999). and references therein.

[3] M. Mezard and G. Parisi, J.Phys.: Condens. Matter 11, A157 (1999) and references therein.

[4] R. Speedy, J. Phys.: Conds. Matter 10, 4185 (1998); ibid. 9, 8591 (1997); ibid. 8, 10907 (1996).

[5] J. Wong and C.A. Angell Glass: Structure by spectroscopy, (Basel: Dekker 1976). J. Brawer Relaxation in Viscous Liquids and Glasses (Columbus, OH: The American Ceramics Society 1985).

[6] C. A. Angell Science 267, 1924 (1995).

[7] K. Binder et al., p. 22 in Complex Behaviour of Glassy Systems, M. Rubí and C. PerezVicente Eds. (Springer Berlin 1997).

[8] A.W. Kauzmann, Chem Rev 43, 219 (1948).

[9] G. Adams and J.H. Gibbs J. Chem. Phys. 43, 139 (1958); J.H. Gibbs and E. A. Di Marzio J. Chem. Phys. 28373 (1958).

[10] M. Goldstein, J. Chem. Phys. 51, 3728 (1969).

[11] E. A. Di Marzio and A.J. Yang, J. Res. NIST 102, 135 (1997) and references therein.

[12] F.H. Stillinger and T.A. Weber, Phys. Rev. A 25, 978 (1982); F.H. Stillinger and T.A. Weber, Science 225, 983 (1984). F. H. Stillinger, Science, 267 1935, (1995).

[13] T.A.Weber and F.H.Stillinger J. Chem. Phys 87, 3252 (1987); F. Sciortino, A. Geiger and H.E. Stanley Nature 354, 218 (1991); F. Sciortino, A. Geiger and H.E. Stanley Phys. Rev. Letts. 65, 3452 (1990).

[14] I..Ohmine, H.Tanaka, P.G.Wolynes J. Chem. Phys 89, 5852 (1988); H. Tanaka, Nature 380, 328 (1996).

[15] S. Sastry, P. G. Debenedetti, and F. H. Stillinger, Nature 393, 554 (1998). See also the related work of H. Jonsson and H.C. Andersen Phys. Rev. Letts. 60, 2295 (1988).

[16] F. Sciortino, S. Sastry and P. Tartaglia cond-mat/9805040

[17] W. Kob, F. Sciortino, and P. Tartaglia, cond-mat/9905090 (1999).

[18] W. Kob and H. C. Andersen, Phys. Rev. E 51, 4626 (1995); Phys. Rev. E 52, 4134 (1995). M. Nauroth and W. Kob, Phys. Rev. E 55, 675 (1997); T. Gleim, W. Kob, K. Binder, Phys. Rev. Lett. 81, 4404 (1998).

[19] S. Nosé, Mol. Phys. 501055 (1983).

[20] In going from Eq.(2) to Eq.(6) we have performed a maximum integrand evaluation of the partition function, a procedure which becomes exact in the thermodynamic limit.

[21] Below $T=0.5, e_{I S}(T)+\frac{3}{2} k_{B} T$ is equal to the average potential energy, supporting the hypothesis that the system mainly probes an harmonic potential landscape.

[22] Y. Rosenfeld and P. Tarazona, Mol. Phys. 95, 141 (1998).

[23] B. Coluzzi, P. Verrocchio, P. Mezard, G. Parisi cond-mat/9904124 (1999).

[24] C.A. Angell, B.E. Richards, and V. Velikov, J .Phys.: Condes. Matter 11, A75 (1999) and references therein.

[25] A. Heuer Phys. Rev. Letts. 78, 4051 (1997). See also for the same model F. H. Stillinger and T. A. Weber, Phys. Rev. A 28, 2408 (1983).

[26] L. Angelani, G. Parisi,G. Ruocco, G. Viliani. Phys. Rev. Letts. 81, 4648 (1998). 
[27] Th. M. Nieuwenhuizen, Phys. Rev. Letts. 80, 5580 (1998); ibid. 79, 1317 (1997). See also cond-mat/9807161, and references therein.

[28] J.-P. Bouchaud, L. F. Cugliandolo, J. Kurchan, and M. Mézard, p. 161-223 in Spin Glasses and Random Fields, Ed.: A.P. Young (World Scientific, Singapore, 1998). L. F. Cugliandolo, J. Kurchan, L. Peliti, Phys. Rev. E 55, 3898 (1997). 


\section{FIGURES}

FIG. 1. (A) Distributions $P\left(e_{I S}, T\right)$ of the $I S$ energy (per atom) for different equilibrium temperatures $T$. From left to right: $T=0.446,0.466,0.5,0.55,0.6,0.8,1.0,2.0,4.0$. (B) $\ln \left[P\left(e_{I S}, T\right)\right]+\beta e_{I S}+C(T)$, for different equilibrium temperatures $T$. Symbols refer to different $T$-values. The curves which do not lay on the continuous line correspond to $T=4.0,2.0,1.5,1.0,0.8$, from bottom to top. The constant $C(T)$ has been chosen to maximize the overlap between curves with different $T$ and the overlap with $S_{\text {conf }}\left(e_{I S}\right)$ (in absolute units) calculated from $\Delta S(T)$, as discussed in the text. $S_{\text {conf }}\left(e_{I S}\right)$ is reported as continuous line both in (B) and in the enlarged $e_{I S}$ range in (C). Note that when $e_{I S}=-7.82, S_{\text {conf }}=0$.

FIG. 2. Disordered-solid (filled squares) and liquid (filled circles) entropies as a function of $T$. Filled rombs are $S_{\text {disordered-solid }}-3 N \ln \left(T / T_{0}\right)$, with $T_{0}=1$, to demonstrate the weakness of the $T$ contribution arising from the $T$-dependence of frequency distribution. Below $T=1$, this weak $T$-dependent contribution is well fitted (long-dashed line) by the quadratic polynomial $3201.62309-402.760885 T+199.228407 T^{2}$, providing a reliable extrapolation of $S_{\text {disordered-solid }}$ to $T$ lower than the studied ones (dotted line). The liquid entropy is extrapolated according to the theoretical predictions discussed in Ref. [22], i.e. $S_{\text {liquid }}+1.5 k_{B} \ln \left(T / T_{0}\right) \sim T^{-2 / 5}$

FIG. 3. Configurational entropy (A) and energy (B) as a function of $T$ calculated as difference of the liquid and disordered-solid entropies and energies, respectively. The arrow indicates $T_{M C T}$

for this system. The full circles in $(\mathrm{B})$ are $\bar{e}_{I S}(T)$. Note that in the region where the harmonic approximation for the disordered solid is expected to be valid [21], $\bar{e}_{I S}(T)$ coincides with the configurational energy. 


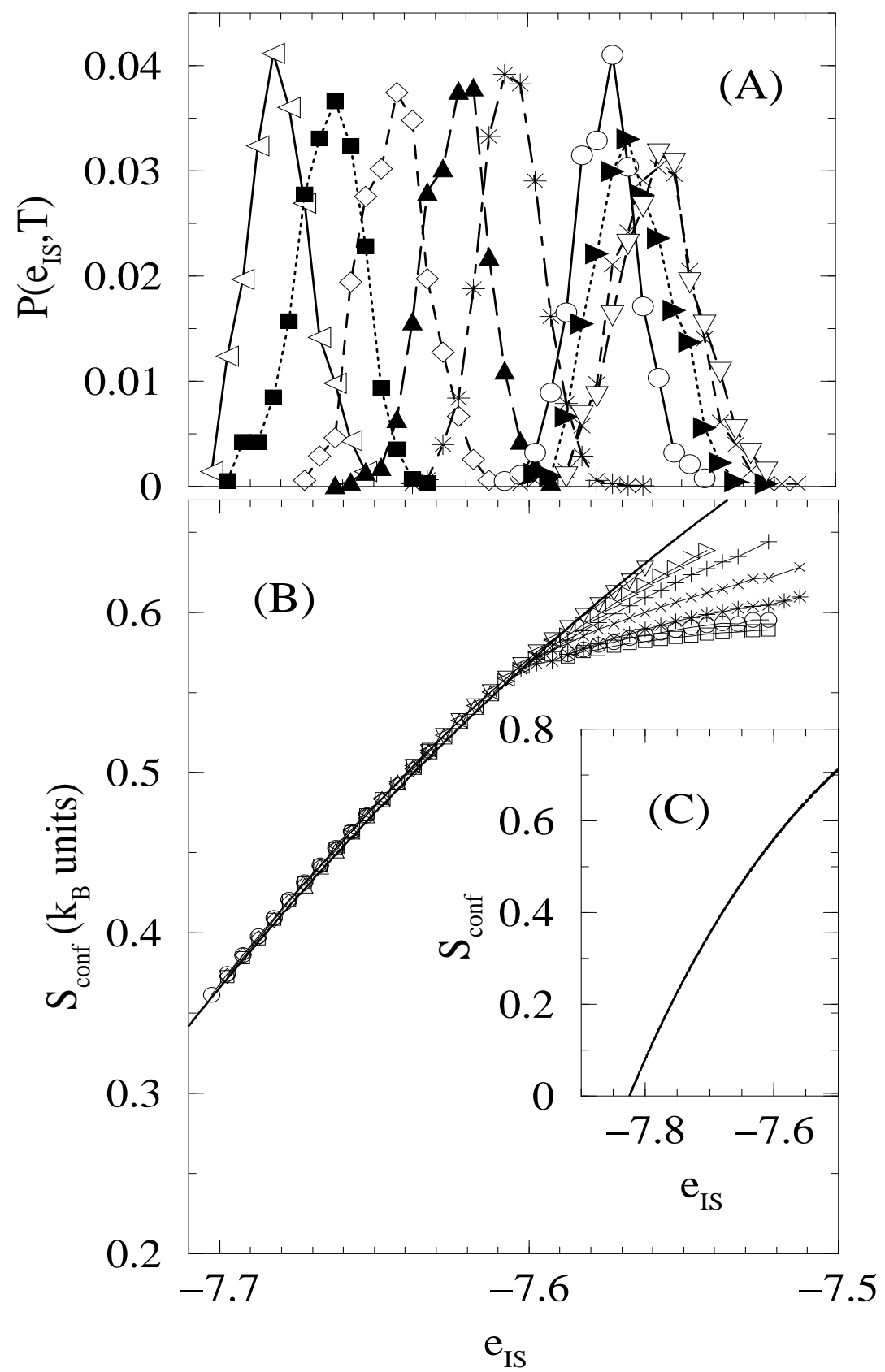

FIG. 1. F. Sciortino et al 


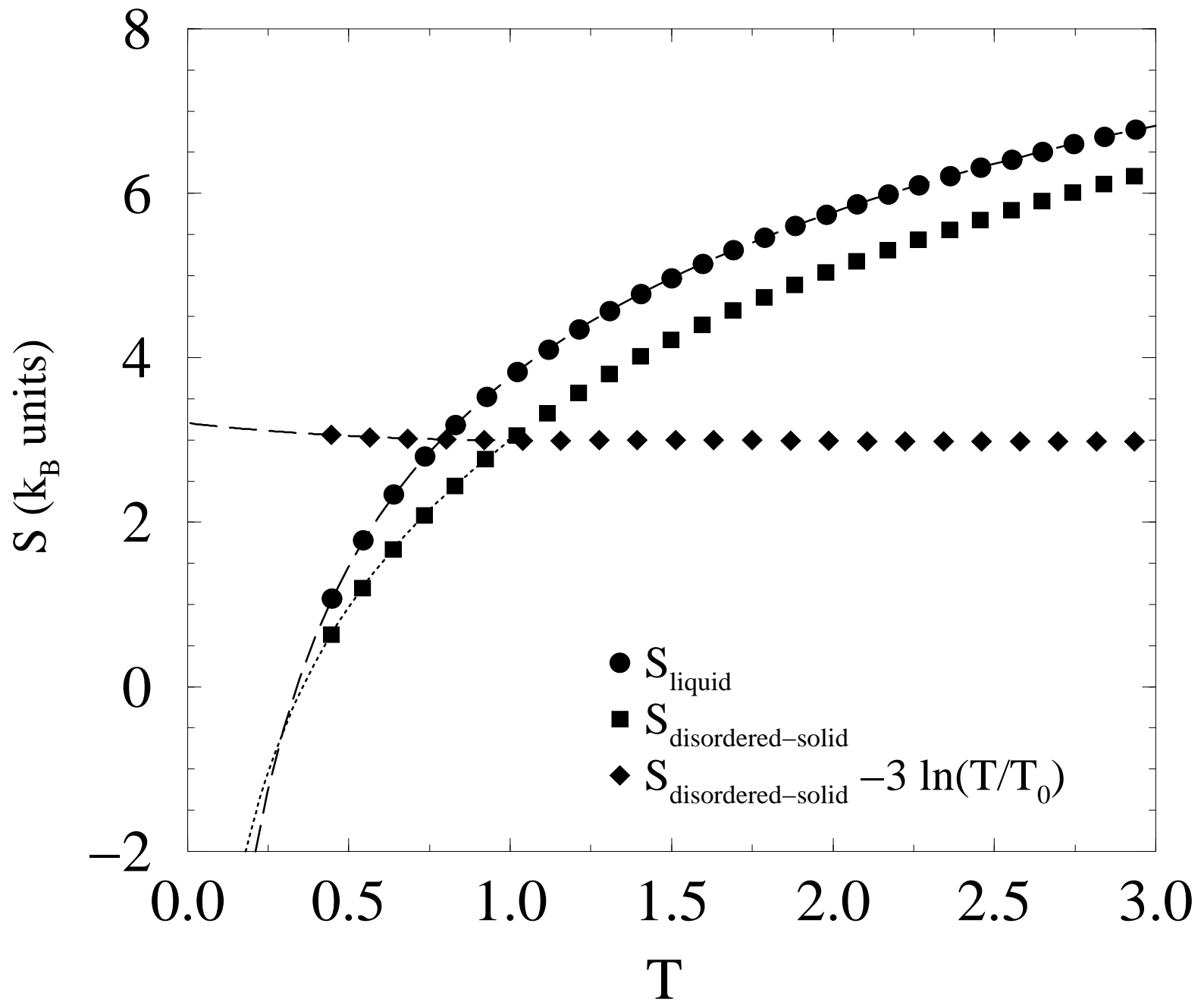

FIG. 2. F. Sciortino et al 


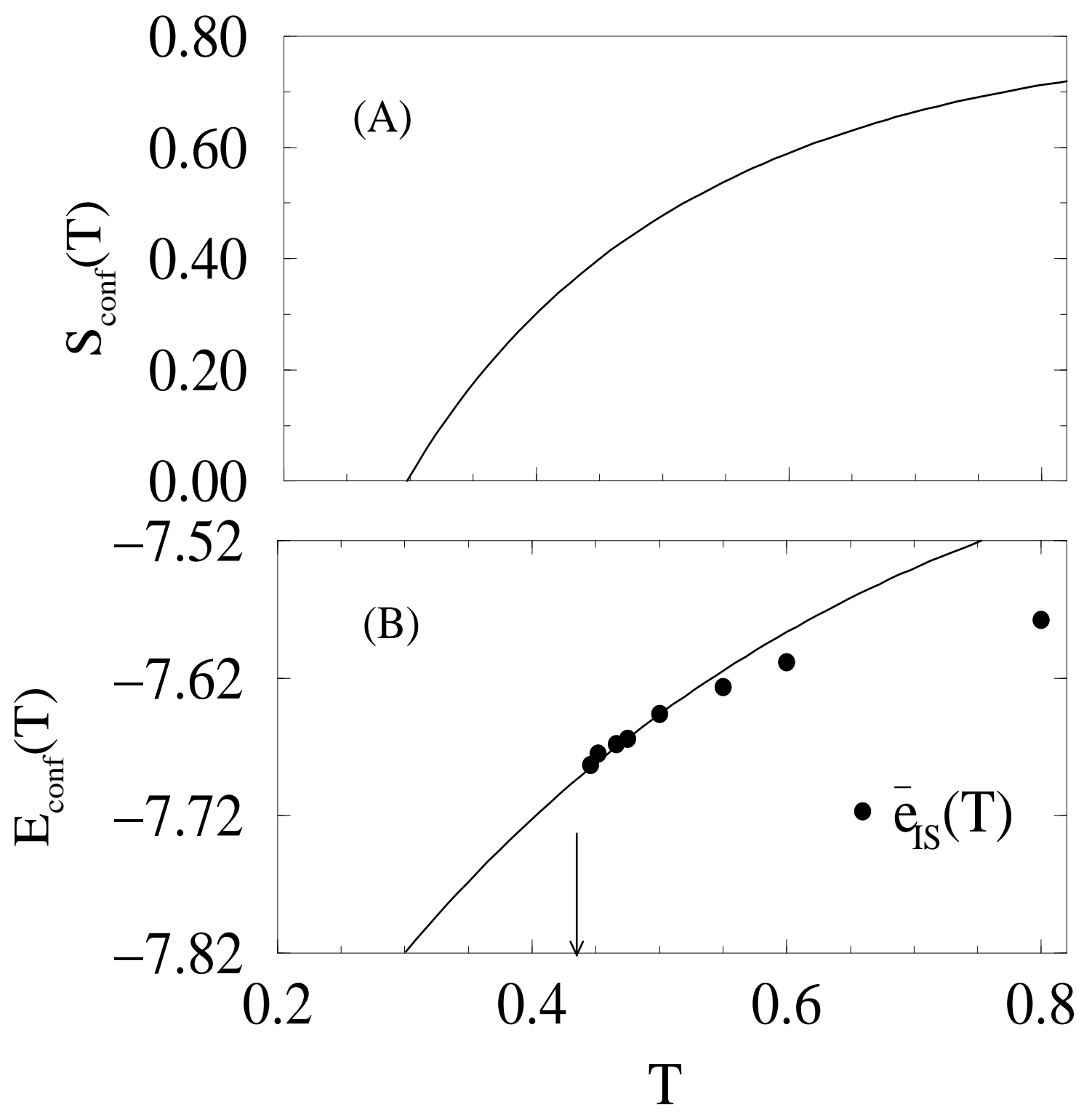

FIG. 3. F. Sciortino et al 